\title{
Isolation of targeting nanobodies against co-opted tumor vasculature
}

\author{
Ilse Roodink', Maarten Franssen', Malou Zuidscherwoude ${ }^{1}$ Kiek Verrijp¹, Tom van der Donk², Jos Raats ${ }^{2}$ and \\ William PJ Leenders ${ }^{1}$
}

Tumor vasculature is in general highly heterogeneous. This characteristic is most prominent in high-grade gliomas, which present with areas of angiogenic growth, next to large areas of diffuse infiltrative growth in which tumor cells thrive on pre-existent brain vasculature. This limits the effectiveness of anti-angiogenic compounds as these will not affect more matured and co-opted vessels. Therefore, additional destruction of existing tumor vasculature may be a promising alternative avenue to effectively deprive tumors from blood. This approach requires the identification of novel tumor vascular targeting agents, which have broad tumor vessel specificities, ie are not restricted to newly formed vessels. Here, we describe the generation of a phage library displaying nanobodies that were cloned from lymphocytes of a Llama which had been immunized with clinical glioma tissue. In vivo biopanning with this library in the orthotopic glioma xenograft models E98 and E434 resulted in the selection of various nanobodies which specifically recognized glioma vessels in corresponding glioma xenografts. Importantly, also nanobodies were isolated which discriminated incorporated pre-existent vessels in highly infiltrative cerebral E434 xenografts from normal brain vessels. Our results suggest that the generation of nanobody-displaying immune phage libraries and subsequent in vivo biopanning in appropriate animal models is a promising approach for the identification of novel vascular targeting agents.

Laboratory Investigation (2010) 90, 61-67; doi:10.1038/labinvest.2009.107; published online 12 October 2009

KEYWORDS: glioma; in vivo biopanning; nanobody; phage display; vascular targeting

High-grade gliomas (WHO grade IV astrocytomas, glioblastoma multiforme $[\mathrm{GBM}])$ are aggressive primary brain tumors that are considered to be among the deadliest of human cancers. These tumors are generally characterized by a necrotic core, surrounded by an angiogenic rim. ${ }^{1,2}$ Importantly, these cancers always present also with large areas of diffuse infiltrative growth, in which tumor cells migrate along white matter tracts or blood vessels. This diffuse infiltrative phenotype strongly limits the effectiveness of surgery and radiotherapy. In addition, the blood-brain barrier in diffuse infiltrative areas is generally intact, contributing to the poor response of these tumors to chemotherapies. $1,3,4$

The occurrence of angiogenesis in GBM has been rationale for extensive testing of angiogenesis inhibitors. However, a number of phase II studies have now revealed that, despite inducing a radiological response, these inhibitors do not prolong overall survival and allow disease progression, ${ }^{5,6}$ likely because diffuse infiltrative growth is angiogenesis independent and provides a mechanism of escape from anti-angiogenic therapies. ${ }^{4-11}$

The phenotypic differences that exist in GBM are reflected in the vasculature, which is highly heterogeneous. ${ }^{12}$ Vessels may be angiogenic as well as more mature or co-opted, and the latter ones may be unsusceptible to anti-angiogenic therapy. ${ }^{13}$ To bypass resistance to anti-angiogenic therapies, additional vascular targeting therapy in which the existing tumor vascular bed is attacked with the aim to acutely deprive tumors from blood supply may be an attractive option. So far, identified targets with specificity for tumor vasculature are restricted to vessels in early stages of maturation. ${ }^{14-16}$ Thus, there is a need for additional tumor vascular targeting agents, which recognize tumor vessels in more mature stages.

One technique that allows for isolation of such targeting agents is biopanning, the procedure of contacting libraries of binding agents with epitopes of interest, and selecting and

\footnotetext{
'Department of Pathology, Radboud University Nijmegen Medical Centre, Nijmegen, The Netherlands and ${ }^{2}$ ModiQuest B.V. and Department of Biomolecular Chemistry, Nijmegen Centre for Molecular Life Sciences, Nijmegen, The Netherlands

Correspondence: I Roodink, MSc, Department of Pathology, Radboud University Nijmegen Medical Centre, PO Box 9101, Nijmegen 6500 HB, The Netherlands. E-mail: I.Roodink@pathol.umcn.nl 
isolating those agents with significant affinity. ${ }^{17}$ In vivo biopannings with peptide phage display libraries have been successfully used to obtain organ-specific vascular targeting peptides in animal models. ${ }^{18,19}$ In addition, we previously provided proof of concept that via in vivo biopanning of phage antibody-display libraries in orthotopic E98 glioma xenografts, ${ }^{11,20}$ antibodies can be isolated that recognize subsets of tumor vessels (submitted). In that work, we used a nanobody phage display library, cloned from lymphocytes of an immune-naive Llama. ${ }^{16}$ Nanobodies are composed of the variable domains of cameloid heavy-chain antibodies, which lack a light chain. As these consist of a single polypeptide chain, nanobodies can be cloned and displayed in a phage context without significant loss of affinity compared with the originating heavy-chain antibody, making them to an attractive class of compounds. ${ }^{21}$ Nanobodies against EGFR have already been successfully used for in vivo tumor targeting purposes. ${ }^{22-24}$

By in vitro biopannings, we previously isolated from our immune-naive library Plexin D1-specific nanobodies with affinities in the range of $10^{-8}-10^{-9} \mathrm{M}$, which suffice for immune stainings, ${ }^{16}$ but are possibly of too low affinity for effective in vivo applications. Matured nanobodies, cloned from immunized animals, will obviously have the advantage of higher affinity. Here, we describe the construction of a phage library, displaying IgG2 and IgG3 isotype-specific nanobodies, which were cloned from lymphocytes after immunization of a Llama glama with a homogenate of surgically obtained GBM. We performed in vivo biopanning of this phage library in the orthotopic glioma xenograft model E98 and the oligodendroglioma xenograft line E434. E98 was used because it displays angiogenic as well as cooptive growth, ${ }^{11}$ whereas E434 is a hallmark example of a xenograft, which exclusively displays diffuse infiltrative growth with no apparent sprouting angiogenesis. ${ }^{11}$ This approach resulted in the isolation of a number of tumor vessel recognizing nanobodies, also against non-angiogenic blood vessels in diffuse infiltrative tumor areas.

\section{MATERIALS AND METHODS Llama Immunization}

All animal experiments were approved by the Animal Experiment Committee of the Nijmegen University and informed consent was obtained from patients with newly diagnosed GBM who did not receive prior treatment, for use of tumor material. A Llama glama was immunized with a homogenate of three surgically obtained clinical GBMs, after confirmation of absence of reactivity in preserum, as determined by immunohistochemical stainings on frozen sections of the same GBMs. GBMs were selected that showed only minimal necrosis, as was determined by H\&E staining. A total of $90 \mathrm{mg}$ glioma tissue was homogenized in $2.5 \mathrm{ml}$ sterile $0.9 \% \mathrm{NaCl}$ solution. The homogenate was aliquoted in $500 \mu \mathrm{l}$ portions and stored at $-80^{\circ} \mathrm{C}$. For primary immunization and boosts, $500 \mu \mathrm{l}$ homogenate was mixed with
$625 \mu \mathrm{l}$ Stimune adjuvant (Cedi Diagnostics, Lelystad, The Netherlands). This suspension was injected intramuscularly into a Llama. Boost immunizations were performed at 28,42 , 70, 98 days after the initial immunization. Serum and blood samples were isolated before each boost and 126 days after the first immunization. At each time point, leukocyte RNA was isolated using the LeukoLOCK Total RNA Isolation System (Ambion, Austin, TX) according to the manufacturer's protocol. In addition, to verify immune responses, $4 \mu \mathrm{m}$ frozen clinical GBM sections were immunostained with the sera obtained at each time point, according to standard protocols. In short, tissues were pre-incubated with normal goat serum to block non-specific binding sites, followed by overnight incubation at $4^{\circ} \mathrm{C}$ with Llama immune serum or pre-immune serum as control. Llama IgG was detected by incubation with horseradish peroxidase-conjugated goat anti-Llama IgG antibody (Bethyl Laboratories Inc, ITK Diagnostics, Uithoorn, The Netherlands). Peroxidase was visualized by the 3-amino-9-ethylcarbazole (ScyTek, Logan, UT) peroxidase reaction and sections were counterstained with haematoxylin.

\section{Llama Phage Display Library Construction}

Llama nanobodies were RT-PCR cloned in phagemid pHENIX as follows. Total leukocyte RNA samples $(2 \mu \mathrm{g})$ isolated on day 28 after the last boost were reverse-transcribed at $42^{\circ} \mathrm{C}$ for $90 \mathrm{~min}$ using Moloney-mouse-leukemia virus reverse transcriptase (Promega, Leiden, The Netherlands). Subsequently, $\mathrm{V}_{\mathrm{HH}}$ antibody fragments were amplified by PCR using Phusion polymerase (Finnzymes, Bioké, Leiden, The Netherlands), forward primer FR1 (5'-GAG GTB CAR CTG CAG GAS TCY GG-3') (PstI restriction site shown in bold italic) and hinge-specific reverse primers LH49 (AAC AGT TAA GCT TCC GCT TGC GGC CGC TGG TTG TGG TTT TGG TGT CTT GGG-3') and SH48 (5'-AAC AGT TAA GCT TCC GCT TGC GGC CGC GGA GCT GGG GTC TTC GCT GTG GTC CG-3') (NotI restriction sites shown in bold), which assured separate amplification of IgG2- and IgG3encoding $\mathrm{V}_{\mathrm{HH}}$ fragments, respectively. ${ }^{25}$ Amplified $\mathrm{V}_{\mathrm{HH}}$ products were agarose gel purified and digested with PstI (New England Biolabs, Westburg, Leusden, The Netherlands) and NotI (New England Biolabs). Fragments were ligated into pHENIX PstI-NotI vector using Quick Ligase (New England Biolabs). This results in $\mathrm{V}_{\mathrm{HH}}$ chimeras with a His8-VSVG-tag at the C-terminus. ${ }^{26}$ The phagemid library was transformed in electrocompetent Escherichia coli TG1 bacteria. Individual clones were picked and analyzed for the presence of full-length insert by standard colony PCR using forward primer pHEN-fw ( $5^{\prime}$-TCA CAC AGG AAA CAG CTA TGA$3^{\prime}$ ) and reverse primer pHEN-rev (5'-GTA ACG ATC TAA AGT TTT GTC G-3'). Full-length insert ratio was determined after running PCR products on a 1.0\% agarose gel.

Phages were grown from this library by infection with the trypsin sensitive helper phage $\mathrm{M} 13 \mathrm{~K} 07^{27}$ and purified from 
the culture supernatant by precipitation with $20 \%$ PEG$2.5 \mathrm{M} \mathrm{NaCl}$ as previously described. ${ }^{28}$

\section{In vivo Selection of Tumor Vessel Binding Nanobodies via Biopanning of Phage Display Libraries}

To grow intracerebral E98 and E434 tumors, Balb/c nude mice were transcranially injected with the glioblastoma xenograft line E98 $(n=2)$ or the oligodendroglioma line E434 $(n=2)$ essentially as described earlier. ${ }^{11}$ In short, a mouse carrying a subcutaneous E98 tumor and one carrying a cerebral E434 xenograft were killed. The tumors were sterile removed, minced to small pieces and filtered through a 70$\mu \mathrm{m}$ mesh nylon filter. The resulting single cell suspensions were subsequently injected through the skull of nude mice under deep anesthesia. After, respectively, 3 and 7 weeks, when mice carrying E98 and E434 xenografts displayed signs of tumor growth (discomfort, weight loss and neurological symptoms), in vivo biopanning was performed as follows: $10^{12} \mathrm{GBM}$ nanobody-phages were intravenously injected. Nanobody-displaying phages were allowed to circulate and bind to their targets for $10 \mathrm{~min}$. Subsequently, mice were anaesthetized using $1.3 \%$ isoflurane $/ \mathrm{N}_{2} \mathrm{O} / \mathrm{O}_{2}$ and cardiac perfusion was performed with $15 \mathrm{ml}$ sterile $0.9 \% \mathrm{NaCl}$ solution to remove unbound phages from the circulation. Mice were then sacrificed by decapitation and brains were collected. A small part was formalin-fixed and paraffin-embedded, the remainder was snap frozen in liquid nitrogen. Paraffin-embedded sections $(4 \mu \mathrm{m})$ were stained with antiM13 p8 antibody (Abcam Limited, Cambridge, UK) to investigate phage distribution in the tumor. In case of E98, tumor areas were dissected from 16 frozen brain sections $(10 \mu \mathrm{m})$ using a laser dissection microscope (Leica AS LMD, Wetzlar, Germany), whereas excessive unaffected brain parenchyma was scratched manually from 18 frozen E434 brain sections $(30 \mu \mathrm{m})$. The remaining $\mathrm{E} 434$ tumor areas were collected in a tube. Phages were eluted from the obtained tumor areas by trypsin treatment $(10 \mathrm{mg} / \mathrm{ml}, 30 \mathrm{~min} \mathrm{RT})$ and infected in $10 \mathrm{ml}$ of log-phase TG1 culture.

Ninety-four randomly selected individual clones were picked and analyzed for the presence of full-length insert as described above. To prevent analysis of duplicate clones, fingerprint analysis was performed by digesting full-length PCR products with the four-cutter enzymes Bst $\mathrm{NI}$ and $B s t \mathrm{U} 1$ followed by electrophoresis on $4 \%$ nusive agarose gels. In addition, these clones were used for small-scale IPTG-induced nanobody production by overnight induction with $1 \mathrm{mM}$ IPTG. Nanobody expression levels in the culture supernatant were determined via dotblot analysis using the mouse monoclonal anti-VSV-G P5D4 and alkaline phosphatase-conjugated rabbit anti-mouse immunoglobulin (Dako, Glostrup, Denmark). Alkaline phosphatase was visualized using nitro blue tetrazolium (NBT; Roche, Almere, The Netherlands) and 5-bromo-4-chloro-3-indolyl phosphate (BCIP; Roche) as substrate. On the basis of the collective data, clones were selected for further analysis by immunohistochemistry. High-level expression of soluble nanobodies was induced in log-phase TG1 cells by culturing at $30^{\circ} \mathrm{C}$ in $2 \times \mathrm{TYA} / 1 \mathrm{mM}$ IPTG. Nanobodies were collected by osmotic lysis as described. ${ }^{16}$

\section{Immunohistochemistry}

Soluble nanobodies, obtained via osmotic lysis of TG1 cells, were tested in immunostaining on $4 \mu \mathrm{m}$ sections of paraffinembedded cerebral mouse xenografts (E98 or E434). After deparaffinization, endogenous peroxidase activity was blocked by incubation with $3 \% \mathrm{H}_{2} \mathrm{O}_{2}$. Antigen retrieval was performed by boiling in $10 \mathrm{mM}$ citrate buffer for $10 \mathrm{~min}$, using a microwave oven set at 180 Watt. Slides were preincubated with normal goat serum to block non-specific binding sites, followed by overnight incubation with nanobodies at $4^{\circ} \mathrm{C}$. Subsequently, detection was performed by the following incubations at room temperature: $1 \mathrm{~h}$ rabbit antiVSV-G antiserum (Sigma-Aldrich, Zwijndrecht, The Netherlands), $30 \mathrm{~min}$ biotinylated anti-rabbit antibody (Vector, Burlingame, CA), and $45 \mathrm{~min}$ avidin-biotin peroxidase complex (Vector). Finally, visualization was performed by the 3-amino-9-ethylcarbazole (ScyTek) peroxidase reaction with haematoxylin as counterstain.

\section{RESULTS \\ Immunohistochemical Evaluation of Llama Immune Response}

Sera obtained at five different time points after initial immunization of a Llama with a homogenate of clinical GBM tissues were tested on the same GBM tissues used for immunization. Whereas preserum was completely negative, after each immunization boost increasing immunoreactivity was seen on tumor cells and extracellular matrix components of the GBMs with optimal responses after the fourth boost (Supplementary Figure 1). Therefore, leukocyte RNA isolated after this boost immunization was used for generation of a nanobody phage display library. The combined IgG2 and IgG3 nanobody library had a size of $10^{7}$ clones of which $88 \%$ contained a full-length insert (data not shown).

\section{In Vivo Selection of Tumor Vessel Binding Phages in Cerebral E98 and E434 Mouse Xenografts}

A suspension of $10^{12}$ phages was intravenously injected in tumor-bearing mice for in vivo biopanning and irrelevant phages were washed from the circulation by cardiac perfusion, after allowing relevant phages to bind for a period of $10 \mathrm{~min}$. Figure 1 shows anti-M13 immunostaining on intracerebral E98 (a) and E434 (b) xenografts. These stainings show a prominent tumor vessel localization of phages (arrowheads in Figure 1a and b). We observed almost no phages associated with normal brain vessels (compare the phage localizations with the anti-CD34 staining of serial sections of E98 and E434 xenografts in Figure 1c and d, respectively). The arrows in Figure $1 \mathrm{c}$ and $\mathrm{d}$ point at vessels, which are not highlighted by anti-M13 staining. 

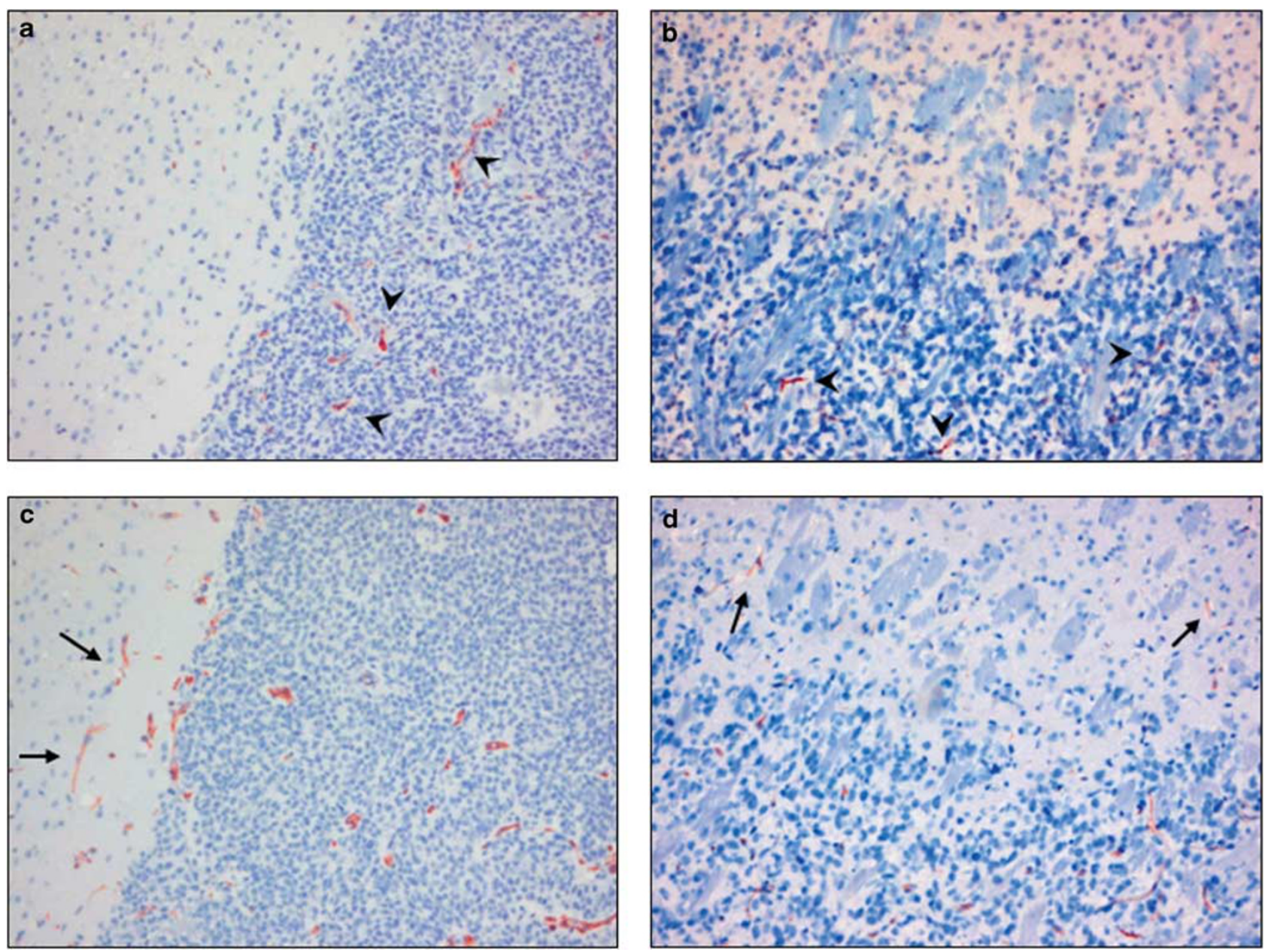

Figure 1 Phage distribution in cerebral glioma xenografts after in vivo biopanning of an immune Llama phage library. Paraffin-embedded sections of E98 (a) and E434 (b) xenografts were analyzed for phage localization by M13 immunostaining. As evidenced by anti-CD34 immunostaining on serial sections of E98 (c) and E434 (d) xenografts phages are clearly associated with the tumor vasculature (arrowheads in $\mathbf{a}$ and $\mathbf{b}$ ), whereas vessels in unaffected brain parenchyma are not highlighted by anti-M13 staining (arrows in $\mathbf{c}$ and $\mathbf{d}$ ). Note the CD34 up-regulation on tumor-associated endothelial cells compared with normal brain capillaries (c and $\mathbf{d}$ ).

Approximately 2800 and 3400 colony-forming phages were rescued from tumor sections from mice carrying E98 and E434 xenografts, respectively.

From the phage populations that were rescued from each xenograft, 94 individual clones were randomly picked for fingerprint analysis and nanobody production. Remaining colonies were collected and pooled and stored as sub-libraries for future rounds of in vivo biopanning. Fingerprint analyses identified 20 and 15 distinct groups of nanobodies, binding to E98 and E434 vasculature, respectively (Table 1). From each group, one clone was selected for nanobody production and immunohistochemical analysis on sections of the corresponding glioma xenografts.

\section{Immunohistochemistry}

As evaluation of vessel staining requires optimal morphology, we performed immunostaining with nanobodies on paraffinembedded sections of cerebral E98 or E434 mouse xenografts.
We accepted here that potentially interesting nanobodies, reactive against epitopes which are lost during fixation, were lost during analyses. Of the 20 nanobodies which were rescued from the E98 biopanning experiments, 15 (75\%) showed positive staining of subsets of tumor vessels in E98 xenografts. Figure 2 shows representative immunostaining with clones obtained from groups A (a) and D (b). Similarly, 8 of the 15 nanobodies (53\%), isolated from in vivo biopanning on E434 xenografts, stained positive on E434 vasculature (Figure $2 \mathrm{c}$ and $\mathrm{d}$ for clones obtained from groups IX and VIII, respectively). As shown in the insets in Figure 2, these nanobodies did not stain vessels in unaffected brain areas.

\section{DISCUSSION}

The diffusely infiltrative growth of human gliomas and the predominantly intact blood-brain barrier in these areas contribute to the poor response of human gliomas to 
Table 1 Percentage E98 and E434 vessel binding clones per restriction pattern of full-length PCR products

\begin{tabular}{|c|c|c|}
\hline $\begin{array}{l}\text { Glioma xenograft } \\
\text { model }\end{array}$ & $\begin{array}{l}\text { Restriction } \\
\text { pattern }\end{array}$ & $\begin{array}{l}\% \text { Clones with } \\
\text { restriction pattern }\end{array}$ \\
\hline \multirow[t]{20}{*}{ E98 } & A & $28 \%$ \\
\hline & B & $5 \%$ \\
\hline & $C$ & $2 \%$ \\
\hline & $\mathrm{D}$ & $5 \%$ \\
\hline & $E$ & $5 \%$ \\
\hline & $\mathrm{F}$ & $3 \%$ \\
\hline & G & $2 \%$ \\
\hline & $\mathrm{H}$ & $2 \%$ \\
\hline & I & $3 \%$ \\
\hline & $J$ & $13 \%$ \\
\hline & K & $11 \%$ \\
\hline & $L$ & $2 \%$ \\
\hline & M & $2 \%$ \\
\hline & $N$ & $3 \%$ \\
\hline & 0 & $3 \%$ \\
\hline & $P$ & $3 \%$ \\
\hline & $\mathrm{Q}$ & $2 \%$ \\
\hline & $\mathrm{R}$ & $2 \%$ \\
\hline & $S$ & $2 \%$ \\
\hline & $\mathrm{T}$ & $3 \%$ \\
\hline \multirow[t]{15}{*}{ E434 } & I & $2 \%$ \\
\hline & ॥ & $12 \%$ \\
\hline & III & $4 \%$ \\
\hline & IV & $14 \%$ \\
\hline & V & $22 \%$ \\
\hline & $\mathrm{Vl}$ & $8 \%$ \\
\hline & VII & $8 \%$ \\
\hline & VIII & $2 \%$ \\
\hline & IX & $8 \%$ \\
\hline & $x$ & $8 \%$ \\
\hline & XI & $2 \%$ \\
\hline & XII & $2 \%$ \\
\hline & XIII & $2 \%$ \\
\hline & XIV & $2 \%$ \\
\hline & $X V$ & $2 \%$ \\
\hline
\end{tabular}

conventional tumor therapies ${ }^{1,3,7}$ and at the same time allow escape from anti-angiogenic therapy ${ }^{5-11,29,30}$ Although gliomas are certainly at the extreme end of a spectrum with respect to infiltrative growth, it was recently shown that targeting of the angiogenic pathway also in subcutaneous xenografts leads to enhanced invasive growth and meta- stases. $^{31,32}$ Such adverse effects are not expected to occur when directing therapy against the existing tumor vasculature. So far, tumor vascular targeting has focused on markers, which are specifically expressed on newly formed vessels, among others the ED-B domain of fibronectin, VCAM- 1 and $\alpha v \beta 3$ integrin. Targeting of newly formed vessels with agents directed against such molecules has already been shown to result in potent anti-tumor effects in preclinical models of subcutaneous xenografts. ${ }^{33-36}$ However, these results cannot be translated directly to clinically relevant situations: in subcutaneous xenografts the vasculature is more or less synchronized and will be homogeneously susceptible to targeting of angiogenic vessels. In contrast, the vasculature of clinical tumors is composed of a heterogeneous population of vessels, ${ }^{12}$ which requires that clinically relevant anti-vascular therapies combine targeting of newly formed vessels with targeting of more mature or co-opted vessels.

The E434 orthotopic glioma model is an extreme example of an angiogenesis-independent tumor, which grows exclusively via incorporation of pre-existent brain vessels, as evidenced by their positivity for glucose transporter-1, which is expressed on brain endothelium with a functional bloodbrain barrier. ${ }^{11}$ Despite a lack of sprouting angiogenesis in these tumors, the vasculature is activated as exemplified by more prominent staining for the vessel marker CD34. ${ }^{11}$ In addition, our data show that these vessels display specific proteins, which are distinct from those expressed on normal vessels, and that these differences in proteome can be used for targeting purposes. The concept of therapeutic targeting of incorporated pre-existent tumor vasculature is probably applicable to all tumors, which grow in vessel dense tissues and which may progress via co-option. Obviously, especially in brain tumors, therapy directed against incorporated pre-existent vessels carries a particular risk of toxicity due to microinfarctions, which may have effects beyond the tumor. This aspect requires thorough examination in relevant tumor models. In other tissues, this issue may be less relevant as some anoxic tissue necrosis may be tolerable, as long as a proper inflammation response can be induced that cleans up the necrotic debris.

In accordance with previous in vivo biopanning experiments in mice carrying cerebral E98 xenografts in our laboratory in which we used an immune-naive nanobody phage display library (submitted) we ended up with nanobodies that specifically reacted with tumor vessels of corresponding cerebral glioma xenografts after only one round of biopanning. We propose that using this in vivo biopanning protocol, phages against common endothelial surface proteins are depleted from the circulation by the excess of normal mouse endothelium. Importantly, following the same in vivo biopanning procedure we rescued six times more interacting phages from E98 xenografts using our immune Llama phage library compared with the immune-naive Llama phage library. This suggests that, as expected, nanobodies against tumor vessel epitopes are more represented in our 

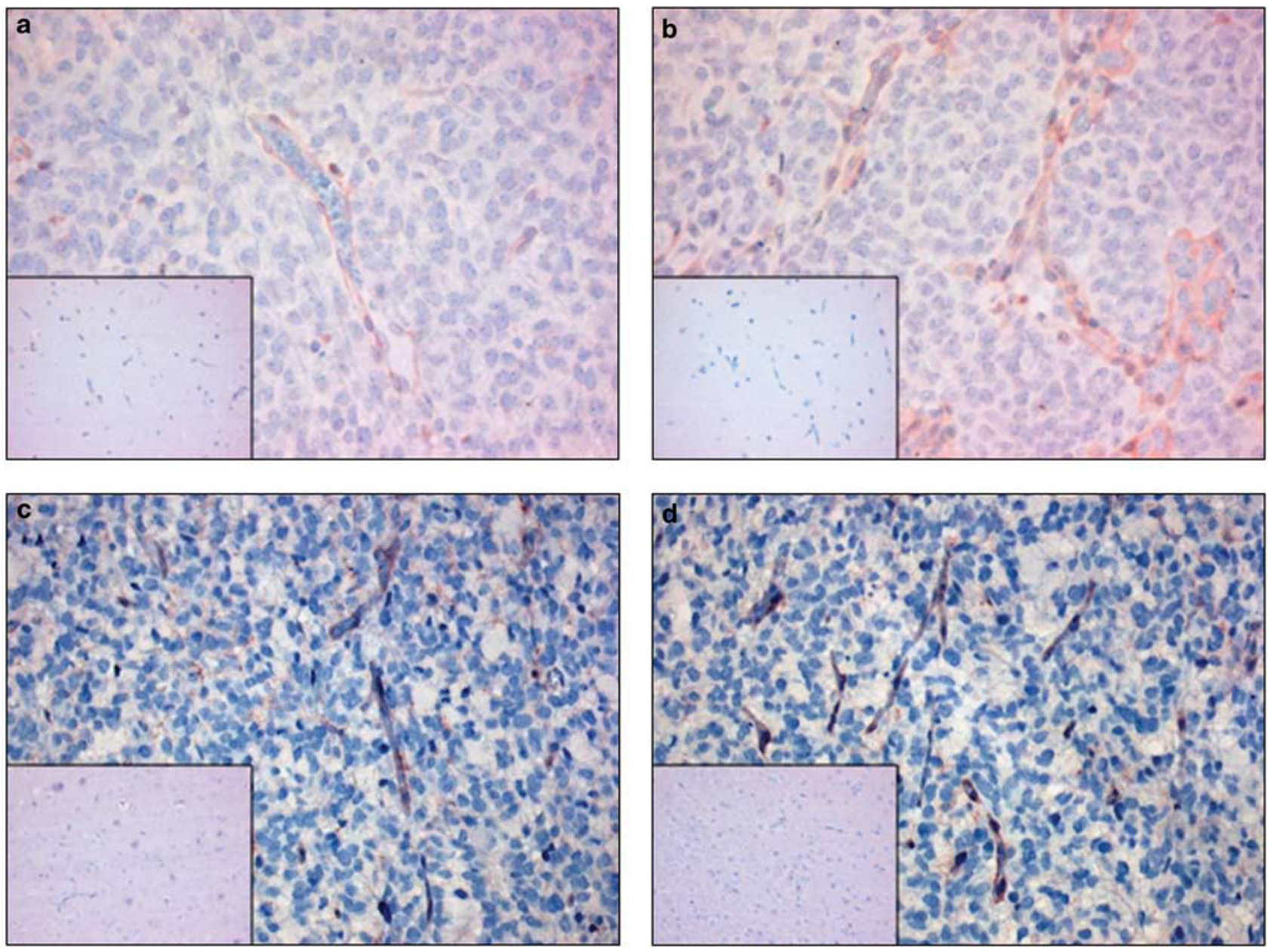

Figure 2 Immunohistochemical analysis of the reactivity of selected nanobodies in glioma xenografts. Clones obtained from groups A (a) and D (b) are representative for nanobodies, which specifically recognized subsets of glioma vessels in immunostaining on paraffin-embedded sections of E98 xenografts. Nanobodies produced by clones from groups IX (c) and VIII (d) show representative immunoreactivity on E434 vasculature. Note the absence of immunoreactivity on vessels in unaffected brain areas (insets).

immune library than in the immune-naive library. In addition, although in vivo biopanning in mouse glioma models resulted in the isolation of nanobodies against mouse endothelium, it is reasonable to assume that they also recognize their human counterparts as they are derived from a Llama after immunization with clinical GBM tissues. Preliminary immunostaining on clinical GBM indeed suggests that this is the case.

The aim of this work was to select nanobodies, which can be further developed into tumor vessel targeting agents. Identification of the ligands, which are recognized by our nanobodies is ongoing in our lab.

In conclusion, we showed that triggering the immune system of a Llama with a homogenate of clinical GBM tissues provoked an immune response against all components present in these tissues. Our phage library-displaying nanobodies, which were cloned from lymphocytes of this Llama is a potential source of targeting agents against different glioma components. Importantly, in vivo biopanning using this phage library in well-characterized mouse glioma models led to selection of various tumor vessel recognizing nanobodies, among them ones, which discriminated incorporated preexistent vessels from normal brain vessels. Overall, construction of nanobody-displaying immune phage libraries and subsequent in vivo biopanning in appropriate animal models is a very promising approach for future identification of novel molecular tools for targeting vascular beds.

Supplementary Information accompanies the paper on the Laboratory Investigation website (http://www.laboratoryinvestigation.org)

\section{ACKNOWLEDGEMENTS}

This work was funded by the Hersenstichting Nederland (grant 14F06(2).03). We are grateful to Conrad van den Broek, Bianca Lemmers-van de Weem and Maikel School for their excellent technical assistance with the animal work. We thank An Claes and Pieter Wesseling for providing E98 and E434 carrying mice.

\section{DISCLOSURE/CONFLICT OF INTEREST}

The authors declare no conflict of interest. 
1. Wesseling P, Ruiter DJ, Burger PC. Angiogenesis in brain tumors; pathobiological and clinical aspects. J Neurooncol 1997;32:253-265.

2. Plate $\mathrm{KH}$, Breier $\mathrm{G}$, Weich $\mathrm{HA}$, et al. Vascular endothelial growth factor is a potential tumour angiogenesis factor in human gliomas in vivo. Nature 1992;359:845-848.

3. Kleihues $P$, Louis DN, Scheithauer BW, et al. The WHO classification of tumors of the nervous system. J Neuropathol Exp Neurol 2002;61:215-225.

4. Claes A, Idema AJ, Wesseling P. Diffuse glioma growth: a guerilla war. Acta Neuropathol 2007;114:443-458.

5. Ali SA, McHayleh WM, Ahmad A, et al. Bevacizumab and irinotecan therapy in glioblastoma multiforme: a series of 13 cases. J Neurosurg 2008;109:268-272.

6. Narayana A, Kelly P, Golfinos J, et al. Antiangiogenic therapy using bevacizumab in recurrent high-grade glioma: impact on local control and patient survival. J Neurosurg 2009:110:173-180.

7. Kunkel $P$, Ulbricht $U$, Bohlen $P$, et al. Inhibition of glioma angiogenesis and growth in vivo by systemic treatment with a monoclonal antibody against vascular endothelial growth factor receptor-2. Cancer Res 2001;61:6624-6628.

8. Rubenstein JL, Kim J, Ozawa T, et al. Anti-VEGF antibody treatment of glioblastoma prolongs survival but results in increased vascular cooption. Neoplasia 2000;2:306-314.

9. Claes $A$, Wesseling $P$, Jeuken $\mathrm{J}$, et al. Antiangiogenic compounds interfere with chemotherapy of brain tumors due to vessel normalization. Mol Cancer Ther 2008;7:71-78.

10. Claes A, Gambarota G, Hamans B, et al. Magnetic resonance imagingbased detection of glial brain tumors in mice after antiangiogenic treatment. Int J Cancer 2008;122:1981-1986.

11. Claes A, Schuuring J, Boots-Sprenger S, et al. Phenotypic and genotypic characterization of orthotopic human glioma models and its relevance for the study of anti-glioma therapy. Brain Pathol 2008;18:423-433.

12. Roodink I, van der LJ, Kusters B, et al. Development of the tumor vascular bed in response to hypoxia-induced VEGF-A differs from that in tumors with constitutive VEGF-A expression. Int J Cancer 2006;119:2054-2062.

13. Leenders WP, Kusters B, Verrijp K, et al. Antiangiogenic therapy of cerebral melanoma metastases results in sustained tumor progression via vessel co-option. Clin Cancer Res 2004;10:6222-6230.

14. Santimaria M, Moscatelli G, Viale GL, et al. Immunoscintigraphic detection of the ED-B domain of fibronectin, a marker of angiogenesis, in patients with cancer. Clin Cancer Res 2003;9:571-579.

15. Castellani P, Borsi L, Carnemolla B, et al. Differentiation between highand low-grade astrocytoma using a human recombinant antibody to the extra domain-B of fibronectin. Am J Pathol 2002;161:1695-1700.

16. Roodink I, Raats J, van der Zwaag B, et al. Plexin D1 expression is induced on tumor vasculature and tumor cells: a novel target for diagnosis and therapy? Cancer Res 2005;65:8317-8323.

17. Hoogenboom HR. Selecting and screening recombinant antibody libraries. Nat Biotechnol 2005;23:1105-1116.

18. Kolonin MG, Sun J, Do KA, et al. Synchronous selection of homing peptides for multiple tissues by in vivo phage display. FASEB $J$ 2006;20:979-981.
19. Rajotte D, Arap W, Hagedorn M, et al. Molecular heterogeneity of the vascular endothelium revealed by in vivo phage display. J Clin Invest 1998;102:430-437.

20. van Kempen LC, Leenders WP. Tumours can adapt to anti-angiogenic therapy depending on the stromal context: lessons from endothelial cell biology. Eur J Cell Biol 2006;85:61-68.

21. Hamers-Casterman C, Atarhouch T, Muyldermans S, et al. Naturally occurring antibodies devoid of light chains. Nature 1993;363:446-448.

22. Huang L, Gainkam LO, Caveliers V, et al. SPECT imaging with 99mTclabeled EGFR-specific nanobody for in vivo monitoring of EGFR expression. Mol Imaging Biol 2008;10:167-175.

23. Cortez-Retamozo V, Backmann N, Senter PD, et al. Efficient cancer therapy with a nanobody-based conjugate. Cancer Res 2004;64: 2853-2857.

24. Tijink BM, Laeremans $T$, Budde $M$, et al. Improved tumor targeting of anti-epidermal growth factor receptor Nanobodies through albumin binding: taking advantage of modular Nanobody technology. Mol Cancer Ther 2008:7:2288-2297.

25. van $\mathrm{KS}$, de $\mathrm{HH}$, de $\mathrm{KP}$, et al. Llama-derived phage display antibodies in the dissection of the human disease oculopharyngeal muscular dystrophy. J Immunol Methods 2003;279:149-161.

26. Kreis TE. Microinjected antibodies against the cytoplasmic domain of vesicular stomatitis virus glycoprotein block its transport to the cell surface. EMBO J 1986;5:931-941.

27. Kristensen P, Winter G. Proteolytic selection for protein folding using filamentous bacteriophages. Fold Des 1998;3:321-328.

28. Raats J, van Bree N, van Woezik J, et al. Generating recombinant anti-idiotypic antibodies for the detection of haptens in solution. $\mathrm{J}$ Immunoassay Immunochem 2003;24:115-146.

29. Kusters B, Leenders WP, Wesseling $P$, et al. Vascular endothelial growth factor-A(165) induces progression of melanoma brain metastases without induction of sprouting angiogenesis. Cancer Res 2002;62: 341-345.

30. Leenders W, Kusters B, Pikkemaat J, et al. Vascular endothelial growth factor-A determines detectability of experimental melanoma brain metastasis in GD-DTPA-enhanced MRI. Int J Cancer 2003;105:437-443.

31. Ebos JM, Lee CR, Cruz-Munoz W, et al. Accelerated metastasis after short-term treatment with a potent inhibitor of tumor angiogenesis. Cancer Cell 2009;15:232-239.

32. Paez-Ribes $M$, Allen $E$, Hudock J, et al. Antiangiogenic therapy elicits malignant progression of tumors to increased local invasion and distant metastasis. Cancer Cell 2009;15:220-231.

33. Dienst $A$, Grunow $A$, Unruh $M$, et al. Specific occlusion of murine and human tumor vasculature by VCAM-1-targeted recombinant fusion proteins. J Natl Cancer Inst 2005;97:733-747.

34. Schraa AJ, Kok RJ, Moorlag HE, et al. Targeting of RGD-modified proteins to tumor vasculature: a pharmacokinetic and cellular distribution study. Int J Cancer 2002;102:469-475.

35. Janssen ML, Oyen WJ, Dijkgraaf I, et al. Tumor targeting with radiolabeled alpha(v)beta(3) integrin binding peptides in a nude mouse model. Cancer Res 2002;62:6146-6151.

36. Dijkgraaf I, Kruijtzer JA, Frielink $C$, et al. Alpha v beta 3 integrintargeting of intraperitoneally growing tumors with a radiolabeled RGD peptide. Int J Cancer 2007;120:605-610. 